\title{
Splitting method for speedy simulation of cascading blackouts
}

\author{
Janghoon Kim, Member IEEE, James A. Bucklew, Fellow IEEE, Ian Dobson, Fellow IEEE
}

\begin{abstract}
Simulation of cascading blackouts poses many challenges, including fast simulation of long series of rare interactions in large grid models. The splitting method advances the simulation by stages, resampling from each stage to advance to the next stage. We apply the splitting method to the simulation of cascading blackouts to efficiently determine the probability distribution of blackout size. Testing on a blackout simulation shows that splitting can quickly compute large blackouts inaccessible to other methods.
\end{abstract}

Index Terms-Simulation, failure analysis, probability, power transmission system reliability

\section{INTRODUCTION}

Reliable electric power transmission is essential to our society and to transforming its energy infrastructure. Many of the new sources and loads transforming our electric energy infrastructure are enabled by the power transmission system and require the high reliability of the current transmission network to be maintained or improved. Also, the grid itself is being transformed towards a "smart grid." In pursuing these transformations, the substantial risks of large, cascading failure blackouts cannot be ignored [1]. A key barrier to quantifying and mitigating these risks is to be able to compute the probability distributions of blackout sizes by running simulations. However, simulating the long, complicated series of rare events in large blackouts is very challenging, and conventional methods of simulation are severely limited by the combinatorial explosion of possible sequences of events and intractable simulation times. In this paper, we extend and apply a simulation method known as splitting that enables rapid and practical computation of large blackout probabilities.

A typical large blackout has an initial disturbance followed by a sequence of cascading events. For example, long and intricate cascades of events caused the August 1996 blackout in Northwestern America [2] that disconnected $30390 \mathrm{MW}$ to 7.5 million customers [3]. The August 2003 blackout in Northeastern America disconnected $61800 \mathrm{MW}$ to 50 million people [4]. The interactions involved are diverse and include deviations in power flows, frequency, and voltage as well as operation or misoperation of protection devices, controls, operator procedures and monitoring and alarm systems [5], [6]. Moreover, the interactions are often rare, unusual, or

J. Kim was with and J.A. Bucklew is with the ECE department, University of Wisconsin, Madison WI 53706 USA, bucklew@engr.wisc.edu. I. Dobson is with the ECpE department, Iowa State University, Ames IA 50011 USA, dobson@iastate.edu. We gratefully acknowledge funding provided in part by the California Energy Commission, Public Interest Energy Research Program. This paper does not necessarily represent the views of the Energy Commission, its employees or the State of California. It has not been approved or disapproved by the Energy Commission nor has the Energy Commission passed upon the accuracy or adequacy of the information. We gratefully acknowledge support in part from DOE grant DE-SC0002283.

(c)IEEE 2012 unanticipated because the likely and anticipated failures are already accounted for in power system design and operation.

Each event in a cascading blackout tends to further weaken and stress the system and make subsequent events more likely. The result is that, although large blackouts are significantly less frequent than small blackouts, they get rarer slowly in a power law fashion ${ }^{1}$ as the blackout size increases [7], [8]. Blackout risk is the product of blackout probability and blackout cost. The importance of the power law in the distribution of blackout size is that larger blackouts become rarer at rate similar to the increase of the cost. This implies that the risk of large blackouts is comparable to, or even exceeding, the risk associated with small blackouts [1]. Thus large blackouts cannot be ignored. However, large blackouts consist of complicated sequences of rare events and their study is strongly limited by simulation speed. For example, to encounter one simulated case of 30 line outages in a 300 bus model of an electrical grid by brute force enumeration may require more than one million cascades to be simulated. Large blackouts often have more than 30 line outages and practical grid models range up to tens of thousands of buses. Many of the compromises currently made in system size and modeling detail can be traced to the lack of fast simulation methods [6], [9].

The idea of splitting is to divide the simulation into stages defined by the number of lines outaged. The simulation starts by sampling a grid operating condition and an initial outage, simulates until either the cascade ends or enough lines outage to reach the second stage, and then saves the state information of the simulation. The simulation of the first stage is repeated until there are enough cases that reach the second stage. We then sample from these cases using the saved state information as starting point and simulate until either the cascade ends or enough lines outage to reach the third stage. The simulation of the second stage is repeated until there are enough cases that reach the third stage. We continue this process in further stages until enough stages are simulated to include the large blackouts of interest.

In this paper, instead of computing the probability of single rare event, we compute the entire probability mass function of the number of lines outaged, since the entire probability mass function is required to assess blackout risk. We show that a straightforward estimator of the probability mass function approximates a maximum likelihood estimator, derive an optimal number of stages for the splitting algorithm, and compare the efficiency of the splitting method to direct simulation.

Cascading blackouts arise from three interacting sources of variation, namely variation in the grid conditions, varia-

\footnotetext{
${ }^{1}$ For example, if blackout size is measured by number of lines outaged, the probability of $n$ lines outaged during a blackout is roughly proportional to $1 / n^{\alpha}$ for a range of $n$, where $\alpha$ is the exponent of the power law.
} 
tion in the initial outages, and variation in the subsequent cascading outages. Therefore to estimate with a simulation the probability mass function of the number of lines outaged we need to sample from each of these sources of variation. Accordingly, each simulated power grid cascade starts with random sampling of the grid conditions and the initial outage, and then continues by sampling the subsequent stages of the cascade. In this paper we focus on the most difficult part of the problem which is the sampling from stages of cascades. The objective of the splitting algorithm is to efficiently obtain the probability mass function of the number of lines outaged.

After an initial test of splitting on a branching process stochastic model that is known to capture some bulk aspects of cascading failure in power systems [10]-[12], we test the splitting methods on the OPA (Oak Ridge-PSerc-Alaska) power systems simulation of cascading blackouts [13]-[15]. OPA represents cascading failure with standard models of the cascading line outages based on simplified power systems physics and operations. The splitting simulation strategy is completely independent of the power system modeling issues, and we emphasize that our goal here is to develop methods that can be used regardless of the actual power system simulation used. That is, OPA functions in this paper only as an indicative test bed, and the splitting method applies generally to any simulation.

\section{Literature REvieW}

Splitting has received much recent interest in the telecommunications literature. A version of this method, called RESTART was introduced in [16] and amplified in various papers [17]-[19] with some elegant probabilistic analysis in [20]. In addition to being used in some communication system simulations, it also has been used in certain physics applications [21], [22]. Optimal choice of splitting stages is addressed in [23].

Wang et al. [24] apply splitting to power system cascading simulation to estimate the probability of a rare event. Based on [23], Wang et al. derive an optimal scheme for choosing levels that minimizes the variance of the event probability. Wang et al. use a simulation of a hidden failure mechanism of cascading failure to compute the probability of the event that more than half the load is shed in the IEEE 118 bus test system with a two stage splitting method. Wang et al. then vary the initial line trip to compute a line trip that has the highest probability of leading to this event. Their hidden failure cascading model probabilistically trips adjacent lines according to their loading. Further work by Shortle on the application of splitting to blackouts, including experience in choosing splitting levels for computing the probability of blackout events, will appear in [25].

Our results in this paper applying splitting to power system simulation were developed in the thesis [26] independently of [24] and focus on estimating the probability distribution of the number of line outages and the performance of splitting for many stages. We test splitting with the OPA power systems simulation of cascading line overloads in which initial line outages are randomly chosen and cascading line outages can either be near or far away from the preceding line outages. The results of both this paper and [24] support the conclusion that splitting is a very promising method for fast and accurate simulation of power system cascading.

There are other approaches to fast simulation used in cascading failure simulations in power systems. For example, Chen et al. [27] use importance sampling [28]-[30] and Kirschen et al. [31] apply correlated sampling and Monte Carlo simulation.

The OPA simulation represents cascading outages and line overloads with a DC load flow model [13], [15]. Varying grid conditions under which the cascades are initiated are generated by sampling from randomly changing patterns of load that keep the total load constant. Cascades are initiated by random line outages, and whenever a line is outaged, the generation and load are redispatched using standard linear programming methods. ${ }^{2}$ The cost function is weighted to avoid load shedding and lines that are limited during the optimization are outaged with a fixed probability. The process of redispatch and testing for outages is iterated until the cascade stops and then the total load shed is recorded.

OPA can also represent the complex systems dynamics of a power system slowly upgrading in response to increasing load and reliability requirements [14], but here we use OPA to simulate a fixed power system. OPA is the simplest cascading line outage blackout model that is based on standard power system physics and operational procedures. The complex system dynamics form of OPA has been validated with data from the Western interconnection of the USA [32].

Branching process models, long applied to other cascading processes [33], [34], have recently been applied to approximate the gross features of cascading blackouts for both simulated data [10] and observed data [11], [12]. Other idealized probabilistic model of cascading failure [35] are well approximated by a branching process [36].

\section{DisCUSSION OF ALTERNATIVE APPROACHES}

Before explaining the splitting method in the next section, we briefly comment on the nature of the simulation problem and some problems with alternative approaches.

We observe that cascading outages of power network generally involve both local and global interactions. For example, the cascade can progress by outages of lines nearby or far from the preceding outages. Since there are many lines that could potentially, albeit with small and varying probability, outage at any stage of a cascade, this implies a huge combinatorial explosion in the number of possibilities that a cascading simulation has to consider and select from. The simulation technique employed must make use of the probabilistic structure of the cascading failure in order to efficiently estimate the risk.

One version of the statistical problem of evaluating blackout risk estimates the probability mass function (pmf) $p(n)$ of the

\footnotetext{
${ }^{2}$ When simulating many outages, as in this paper, islanding can occur. Since OPA implements line outages by making both the line reactance high and enforcing zero line flow [13], the generator redispatch and load shed optimization used in OPA will shed load as needed both in the entire grid when it is connected and in each island when it is islanded.
} 
number of line outages. A first approach to this challenging problem would be a direct Monte Carlo simulation. After simulating an initial disturbance, one uses power system modeling coupled with a probabilistic model for line outages to simulate the power system. Each simulation produces a certain number of line outages. We then count the number of times we see $n$ line outages and divide by the total number of simulations to get an estimate, $\hat{p}(n)$.

There are several important problems with such a naive approach. The vast majority of simulations will terminate with very few lines outaged. Thus we are heavily sampling from the most likely parts of the distribution. For example, we may have ninety five percent of our samples falling in the range of zero to five line outages. While our estimates for the pmf will be excellent for its first 5 or so values, our estimates for the more unlikely higher number of lines (but of greater interest due to their higher impact) will likely be very poor indeed. For example, OPA simulation of the IEEE 300 bus test system would need more than a year to get 100 samples of more than 30 line failures when a Pentium Dual Core $3.0 \mathrm{GHz}$ computer is used.

A second possible approach is to use importance sampling [37] to drive the simulation deeper into the tree of possibilities. However, this approach also has problems. Experience has shown that choosing the sampling distribution must be done with great care. It is easy enough to "over" bias and obtain samples deep into the tree; however, the variance associated with such procedures can easily be greater than the variance of not using importance sampling at all and proceeding with the direct Monte Carlo. In reality different biasing would be required for every possible value desired in the pmf, thus leading to a simulation strategy of great complexity and inefficiency. Furthermore, we should note that there is a long history of observations in the importance sampling community over the difficulty of designing good biasing schemes for simulations of long cascading trees. It is a very difficult problem that is still very much unresolved.

\section{SPLITTING}

We use a splitting technique [38], [39] to perform the simulation. The splitting method simulates the cascades in stages defined by numbers of line outages. Suppose there are $L$ splitting stages, with $0=S_{0}<S_{1}<S_{2}, \ldots, S_{L-1}<S_{L}=$ $M$ line outages respectively, as shown in Figure 1. The stage one simulation starts by sampling a grid operating condition and an initial disturbance and then simulates until we have a "sufficient" number of trajectories, denoted as $K_{1}$, that have greater than $S_{1}$ line outages. We denote $N_{1}$ as the total number of simulations necessary to achieve these $K_{1}$ trajectories. The $K_{1}$ trajectories will make up our empirical entry distribution of trajectories that have greater than $S_{1}$ line outages. Define $N_{1}(k)$ as the number of trajectories in the stage one simulation that have exactly $k$ lines outaged for $k=1,2, \ldots, S_{1}$. Define $\hat{p}(k)$ as the relative frequency estimator of the probability of $k$ outages. Then

$$
\hat{p}(k)=N_{1}(k) / N_{1}, \quad 1 \leq k \leq S_{1} .
$$

Define $A(k)$ as the event that there are greater than $k$ outages. Then the estimated probability of $A\left(S_{1}\right)$ is

$$
\hat{P}\left[A\left(S_{1}\right)\right]=\frac{K_{1}}{N_{1}} .
$$

We now view the $K_{1}$ trajectories of the cascading process that reached $A\left(S_{1}\right)$ as an empirical distribution from which to begin the next stage of the simulation. That is, these states form an empirical distribution over the ensemble of all trajectories that enter the set $A\left(S_{1}\right)$.

Now, sampling randomly from those $K_{1}$ points, we simulate trajectories until either the trajectory stops or reaches greater than $S_{2}$ lines outaged. We continue simulating (say it requires $N_{2}$ times) until we have $K_{2}$ trajectories with greater than $S_{2}$ lines outaged. Define $N_{2}(k)=$ number of trajectories in the stage two simulation that have exactly $k$ lines outaged for $k=S_{1}+1, S_{1}+2, \ldots, S_{2}$. Then

$$
\hat{p}\left(k \mid A\left(S_{1}\right)\right)=\frac{N_{2}(k)}{N_{2}}
$$

and

$$
\hat{p}(k)=\hat{p}\left(k \mid A\left(S_{1}\right)\right) \hat{P}\left(A\left(S_{1}\right)\right)=\frac{K_{1}}{N_{1}} \frac{N_{2}(k)}{N_{2}}, S_{1}<k \leq S_{2} .
$$

$A\left(S_{2}\right)$ is the event that there are greater than $S_{2}$ outages. Then the estimated probability of $A\left(S_{2}\right)$ given that $S_{1}$ occurred is

$$
\hat{P}\left[A\left(S_{2}\right) \mid A\left(S_{1}\right)\right]=\frac{K_{2}}{N_{2}}
$$

and, since $S_{1}<S_{2}, A\left(S_{1}\right) \supset A\left(S_{2}\right)$ and

$$
\begin{aligned}
\hat{P}\left[A\left(S_{2}\right)\right] & =\hat{P}\left[A\left(S_{2}\right) \cap A\left(S_{1}\right)\right] \\
& =\hat{P}\left[A\left(S_{2}\right) \mid A\left(S_{1}\right)\right] \hat{P}\left[A\left(S_{1}\right)\right]=\frac{K_{1}}{N_{1}} \frac{K_{2}}{N_{2}} .
\end{aligned}
$$

The $K_{2}$ trajectories that have greater than $S_{2}$ lines outaged then form an empirical distribution. That is, these states form an empirical distribution over the ensemble of all trajectories that enter the set $A\left(S_{2}\right)$ given that they have already occurred in $A\left(S_{1}\right)$. We continue this process for the successive stages $3,4, \ldots, L$. For $S_{i-1}<k \leq S_{i}$,

$$
\hat{p}(k)=\frac{K_{1}}{N_{1}} \frac{K_{2}}{N_{2}} \ldots \frac{K_{i-1}}{N_{i-1}} \frac{N_{i}(k)}{N_{i}} .
$$

One of the most attractive features of splitting is that we can efficiently generate samples of the whole probability mass function as we move out over these successive stages.

The data that we collect during the simulation consists of

$$
\begin{array}{ll}
N_{i}, & i=1, \ldots, L, \\
N_{i}(k), & S_{i-1}<k \leq S_{i}, i=1, \ldots, L, \\
K_{i}, & i=1, \ldots, L .
\end{array}
$$

From the simulation data (8), we would like to compute the maximum likelihood estimators $\hat{p}(k), k=1,2, \ldots, M$, for the probability mass function of the number of lines outaged. It appears to be very difficult to find the precise likelihood function for the data (8). If, however, we assume that at stage $i$, the number of points $K_{i}$ that make up the empirical entry distribution is large enough to allow the next 


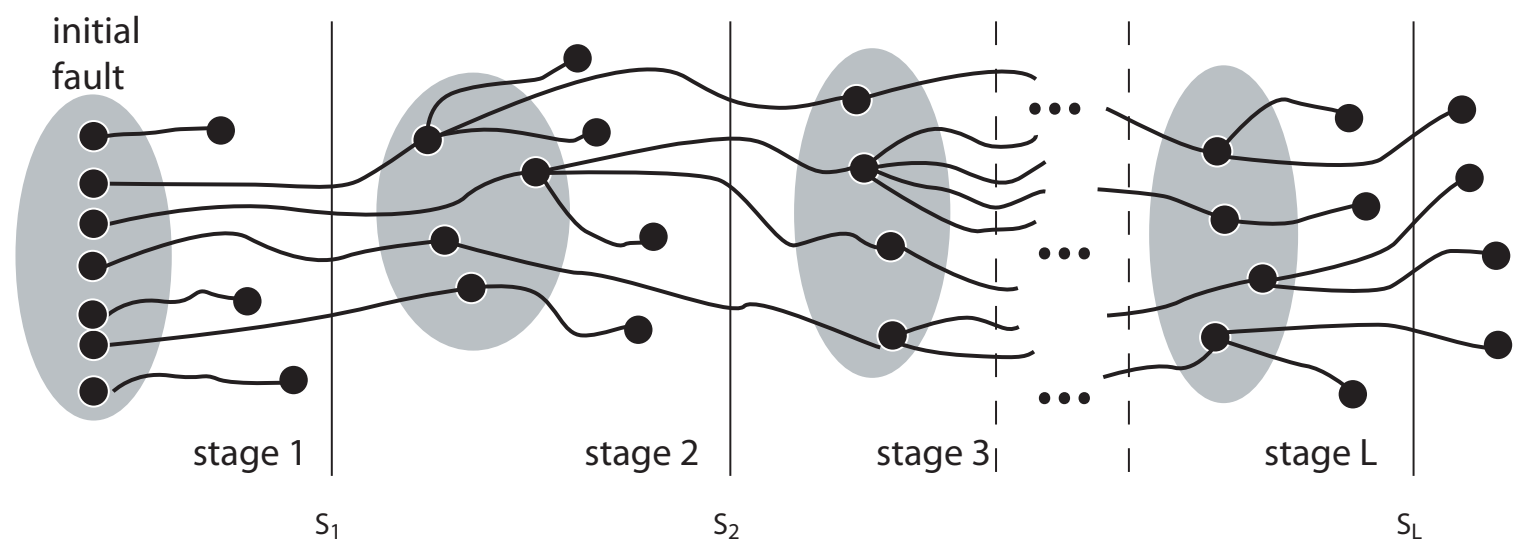

Fig. 1. Splitting method trajectories and stages. Sampling distributions for each stage are shown in gray. In this figure, $S_{1}=S_{2}=\cdots=4, K_{1}=K_{2}=$ $\cdots=4, N_{1}=7, N_{2}=8, N_{3}=8, \ldots, N_{L}=7$.

stage simulation to proceed as if it were being sampled from the true entry distribution, we may easily write down the likelihood function using multinomial probabilities. Thus, the approximate likelihood function is

$$
\prod_{l=1}^{L} \frac{N_{l} !}{K_{l} ! \prod_{\substack{k=\\ S_{l-1}+1}}^{S_{l}(k) !}}\left(\frac{\gamma\left(S_{l}\right)}{\gamma\left(S_{l-1}\right)}\right)^{K_{l}} \prod_{\substack{k==\\ S_{l-1}+1}}^{S_{l}}\left(\frac{p(k)}{\gamma\left(S_{l-1}\right)}\right)^{N_{l}(k)}
$$

where

$$
\gamma(s)=P[\text { number of outages }>s]=1-\sum_{j=1}^{s} p(j) .
$$

Note that since $S_{0}=0, \gamma\left(S_{0}\right)=1$. Since $N_{l}=K_{l}+$ $N_{l}\left(S_{l-1}+1\right)+\ldots+N_{l}\left(S_{l}\right)$, the approximate likelihood function (9) becomes

$$
C \prod_{l=1}^{L} \gamma\left(S_{l}\right)^{K_{l}} \gamma\left(S_{l-1}\right)^{-N_{l}} \prod_{\substack{k=\\ S_{l-1}+1}}^{S_{l}} p(k)^{N_{l}(k)},
$$

where $C$ is a constant. As we will see, the values of $p(k)$ for $k=1,2, \ldots, M$ that maximize (11) are precisely the maximum likelihood estimates. Taking the logarithm of (11) and neglecting constant terms, our estimation problem for $p(k)$ is to maximize

$$
\begin{aligned}
Z=\sum_{l=1}^{L}\left[K_{l} \log \gamma\left(S_{l}\right)-\right. & N_{l} \log \gamma\left(S_{l-1}\right) \\
& \left.+\sum_{k=S_{l-1}+1}^{S_{l}} N_{l}(k) \log p(k)\right] .
\end{aligned}
$$

We have

$$
\gamma\left(S_{l}\right)=1-\sum_{j=1}^{S_{l}} p(j)=\frac{K_{1} K_{2} \ldots K_{l}}{N_{1} N_{2} \ldots N_{l}},
$$

so that

$$
\gamma\left(S_{l}\right)=\gamma\left(S_{l-1}\right) \frac{K_{l}}{N_{l}}
$$

Then, for $S_{i-1}<k \leq S_{i}$,

$$
\begin{aligned}
\frac{\partial Z}{\partial p(k)} & =\sum_{l=i}^{L} \frac{-K_{l}}{\gamma\left(S_{l}\right)}+\sum_{l=i+1}^{L} \frac{N_{l}}{\gamma\left(S_{l-1}\right)}+\frac{N_{i}(k)}{p(k)} \\
& =\frac{-K_{i}}{\gamma\left(S_{l}\right)}+\frac{N_{i}(k)}{p(k)},
\end{aligned}
$$

which vanishes when $p(k)$ is the straightforward estimator (7).

\section{Designing optimal NUMBer of STAGES}

For given maximum target number of failures $M$, it is known [23] that when the number of stages $L$ is given, the optimum choice of stages $S_{1}, S_{2}, \ldots, S_{L}$ is given by identical probabilities of reaching next stage so that $\gamma\left(S_{L} \mid S_{L-1}\right)=$ $\ldots=\gamma\left(S_{1} \mid S_{0}\right)$ and the number of simulation in each stage is the same so that $N_{1}=N_{2}=\ldots=N_{L}$. This section gives a way to choose an optimal number of stages $L$. Letting $\gamma=\gamma\left(S_{L}\right)$ be the probability of reaching final stage $S_{L}=M$ from the start, our goal is to find the optimal $L$ that minimizes the variance of the estimate $\hat{\gamma}$.

It is known from [23] that

$$
\sigma^{2}(\gamma)=\sigma^{2}\left(\gamma\left(S_{1} \mid S_{0}\right) \ldots \gamma\left(S_{L} \mid S_{L-1}\right)\right) \approx \gamma^{2} \sum_{i=1}^{L} \frac{1}{K_{i}}
$$

$\gamma=\gamma\left(S_{L}\right)$ is a fixed constant since $M$ is given. We minimize the total number of simulations when the variance of estimated probability $\sigma^{2}(\gamma)$ is a constant $v$ :

$$
\text { minimize } \sum_{i=1}^{L} N_{i}=L N \text { subject to } \sum_{i=1}^{L} \frac{1}{K_{i}}=v \text {. }
$$

Since $K_{i}=N_{i} \gamma\left(S_{i+1} \mid S_{i}\right)=N \gamma^{\frac{1}{L}}$, the constraint becomes $L=K v=N \gamma^{\frac{1}{L}} v$ and

$$
L N=\frac{L^{2}}{v \gamma^{\frac{1}{L}}} .
$$

Differentiating (13) with respect to $L$ gives

$$
\frac{1}{v \gamma^{\frac{1}{L}}}(2 L+\ln \gamma)
$$

so that $L=-\frac{1}{2} \ln \gamma$, rounded to the nearest integer, is the optimal number of stages. 


\section{EFFICIENCY OF SPLITTING METHOD}

To compare the efficiency of both methods, we analyze how many samples the splitting method and direct method need to get the same variance of probability estimates for both methods. Before the first splitting, when $0<k \leq S_{1}$, there is no difference between the splitting and direct methods. Therefore we will consider $S_{\ell-1}<k \leq S_{\ell}$ for $\ell \geq 2$.

For the direct method, the number of occurrences of the event of $k$ outages in $n$ samples is binomial, and the variance of the estimated probability is

$$
\sigma^{2}(p(k))=\frac{p(k)(1-p(k))}{n} \approx \frac{p(k)}{n}
$$

for $p(k)<<1$. Since the true value of $p(k)$ is unknown, the estimated empirical $\hat{p}(k)$ is used instead as an approximation.

For the splitting method, we assume that the design of all the stages is optimal as in section $\mathrm{V}$. Then $K_{1}=K_{2}=\ldots=K_{\ell}$ are the same constant $K$, and the expected number of simulations $N_{1}=N_{2}=\ldots=N_{\ell}$ are the same constant $N$. We write $c=K / N$. The design entails that $K>>1$ and $N_{l}(k)>>1$. And, although it is up to design, one can readily select the stages so that $\gamma\left(S_{l+1} \mid S_{l}\right)<<1$ and $N_{l}(k) / N_{l}<<1$. Then the estimator of $p(k)$ becomes

$$
\hat{p}(k)=\left(\frac{K}{N}\right)^{\ell-1} \frac{N_{\ell}(k)}{N_{\ell}}=c^{\ell-1} \frac{N_{\ell}(k)}{N_{\ell}} .
$$

Recall that [23] derived expression (12) for $\sigma^{2}(\gamma)$ when $K_{i}>>1$ and $\gamma\left(S_{i+1} \mid S_{i}\right)<<1$ for all $i$. A similar calculation yields

$$
\begin{aligned}
\sigma^{2}(p(k)) & =\sigma^{2}\left(\gamma\left(S_{1} \mid S_{0}\right) \ldots \gamma\left(S_{\ell-1} \mid S_{\ell-2}\right) \frac{N_{\ell}(k)}{N_{\ell}}\right. \\
& \approx(p(k))^{2}\left(\frac{\ell-1}{K}+\frac{1}{N_{\ell}(k)}\right),
\end{aligned}
$$

when $N_{\ell}(k)>>1$ and $\frac{N_{\ell}(k)}{N_{\ell}}<<1$ in addition to $K>>1$ and $\gamma\left(S_{i+1} \mid S_{i}\right)=\gamma<<1$ for all related $i$.

The splitting method has $\ell N$ samples, so the ratio $R$ of the number of splitting method samples to the number of direct method samples is $R=\ell N / n$. Small $R$ indicates a better relative efficiency of the splitting method.

If both methods give the same variance, equating (15) and (18), and substituting for $p(k)$ from (16) yields

$$
R=\frac{\ell N}{n}=\ell\left(\frac{(\ell-1) N_{\ell}(k)}{K}+1\right) c^{\ell-1} .
$$

Since $N_{\ell}(k) / K \leq N / K=c^{-1}>1$, we can obtain the bound

$$
R \leq \ell^{2} c^{\ell-2} .
$$

Since $c<<1$, the bound (20) shows a large relative efficiency of the splitting method of estimating $p(k)$ when the number of splitting stages $\ell$ to reach $k$ outages is moderate or large.

The total computing time of the simulation is most influenced in a straightforward way by the number of cascades sampled. The total computing time is also affected by the average cascade length and the overhead for each cascade sampled. The splitting method cascade samples are limited to one splitting stage, and therefore are on average faster to compute than the unlimited cascade samples of the direct method. However, each splitting method cascade sample that reaches the next stage must save the entire system state in addition to the diagnostics of the cascading, and this additional overhead takes some simulation time.

\section{TESTING ON A BRANCHING PROCESS}

We use splitting to evaluate the probability mass function of the total number of progeny of a simple branching process. Branching processes have advantages of simplicity and analytic tractability that allow us to develop splitting methods on branching processes before applying these methods to a power system blackout simulation.

The Galton-Watson branching process model [33], [34] gives a way to quantify the propagation of cascading failures with a propagation parameter $\lambda$. In the Galton-Watson branching process, the failures are regarded as produced in stages. Stage 0 has one initiating failure. The failures in each stage independently produce further failures in the next stage according to a probability distribution called the offspring distribution with mean $\lambda$. We are ultimately interested in computing the probability mass function, $p(n)=$ $P$ (number of lines outaged $=n$ ) for values of $n$ from one to some large number. Equivalently, we compute the complementary cumulative probability distribution or survival function, $S(n)=P($ number of lines outaged $>n)$ for this same range of $n$. In the setting of a Galton-Watson branching process we have, in some cases, an analytic expression for $p(n)$ and we can compare our simulation estimates to the "gold standard" of the true value. Since, these probabilities can vary several orders of magnitude over the ranges of $n$ that we use, we will compare splitting with direct simulation by computing and comparing the relative error $R(n)$, where

$$
R(n)=\frac{S(n)-\hat{S}(n)}{S(n)}
$$

and $S(n)$ is the true value of the survival function and $\hat{S}(n)$ is the Monte-Carlo estimate produced by either a splitting or direct simulation.

In Figures 2 and 3 we see the relative error $R(n)$ in computing the survival function of the number of lines outaged from one to one hundred lines via direct and splitting simulations for a Galton-Watson branching process with Poisson offspring distribution with mean value $\lambda=0.7$. (In this case, the total number of lines outaged follow a Borel distribution, which means that we can compute $S(n)$ analytically.) To obtain simulations that have relative errors even on the order of one third, we found that we needed about 12 million direct simulations with an elapsed time on a laboratory workstation of 50,000 seconds. Using splitting, we can produce a better relative error with 7600 simulations and an elapsed time of little more than 26 seconds; that is, a speedup of over 3 orders of magnitude. 


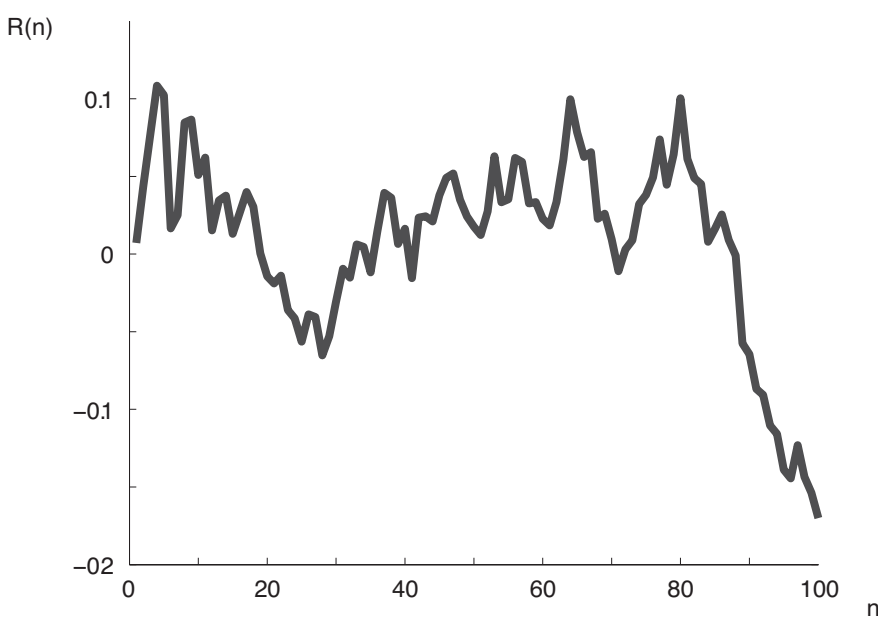

Fig. 2. Relative error $\mathrm{R}(\mathrm{n})$ versus $\mathrm{n}$ to show performance of direct simulation of a branching process (12000000 runs). Mean offspring propagation $\lambda=0.7$.

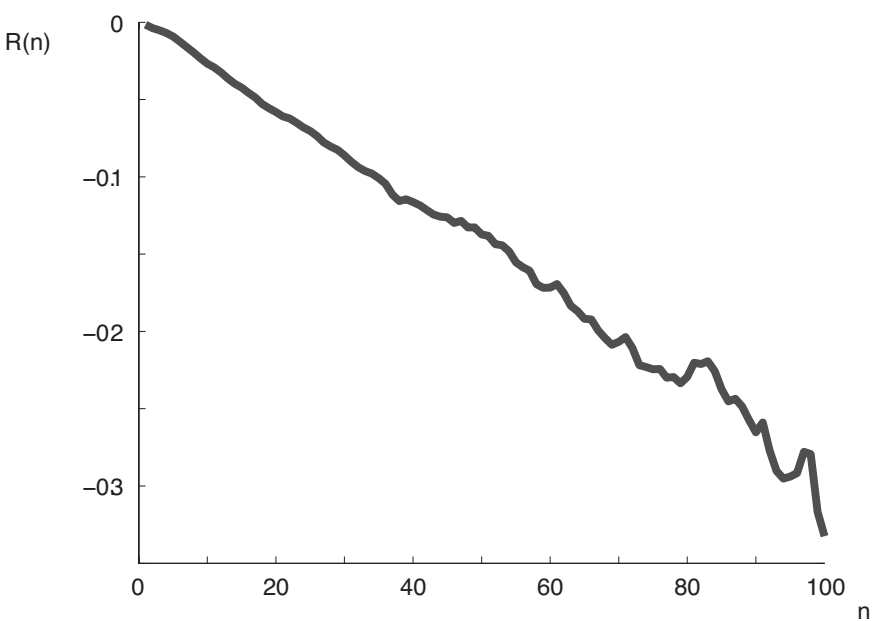

Fig. 3. Relative error $\mathrm{R}(\mathrm{n})$ versus $\mathrm{n}$ to show performance of splitting simulation of a branching process (7600 runs). Mean offspring propagation $\lambda=0.7$.

\section{TESTING ON OPA}

We test the performance of the splitting method with the OPA simulation on the IEEE 118 bus and 225 bus systems. ${ }^{3}$

\section{A. Simulating to reach 12 line outages with 3 stages}

We first check that the splitting method gives the same probability estimates as the direct method using the IEEE 118 bus system. A large number of cascades are simulated to obtain good accuracy.

For the splitting method, we choose three stages $L=3$ with $S_{0}=0, S_{1}=4, S_{2}=8, S_{3}=12$ line outages. We simulate 50000 cascades to obtain $N_{1}=4900$ nonzero cascade samples for stage $S_{1}=4, N_{2}=40000$ times for stage $S_{2}=8$, and $N_{3}=30000$ times for stage $S_{3}=12$. The total number of samples of cascade stages is 120000 .

${ }^{3}$ The parameters of OPA are: $p_{0}$ is 0.001 and $p_{1}$ is 0.15 . Generation margin is 0.3 and $\kappa$ is 0.04 . The load level is 1.0 and the daily load fluctuation is 1.2. There is no upgrade of lines or generators.

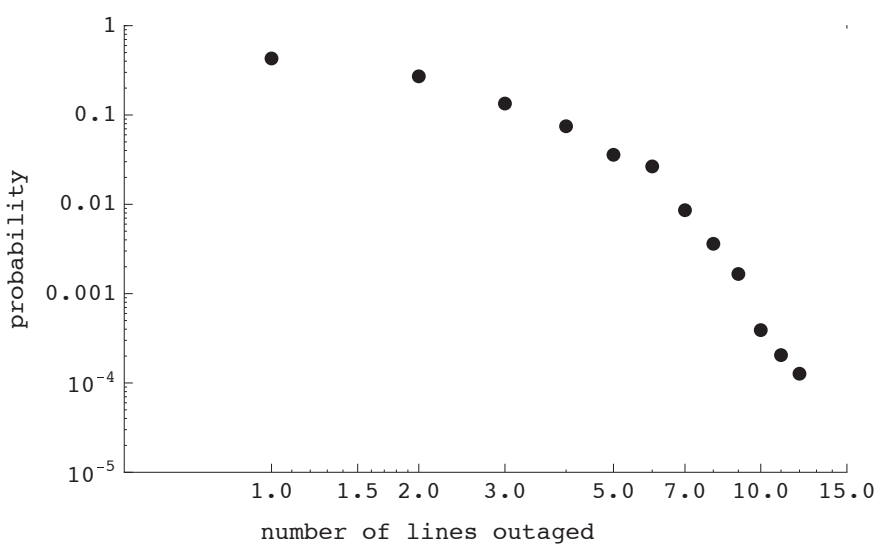

Fig. 4. Probability distribution of the number of line outages using the splitting method on the IEEE 118 bus system.

For the direct method, we simulate 200000 samples of cascades and obtain from these $n=38000$ nonzero cascade samples in which there is at least one line outage.

Table I shows the probability distribution of the total number of line outages from the two methods. And Fig. 4 shows the probability distribution of the total number of line outages from the splitting method. The distribution from the direct method is empirical, and the distribution from the splitting method is calculated using the estimator (7). Both methods show similar distributions.

TABLE I

DISTRIBUTION OF THE NUMBER OF OUTAGES FOR THE TWO METHODS

\begin{tabular}{cccc} 
failures & direct & splitting & standard deviation \\
\hline 1 & 0.4406 & 0.4296 & 0.003 \\
2 & 0.2816 & 0.2774 & 0.003 \\
3 & 0.1194 & 0.1375 & 0.002 \\
4 & 0.0675 & 0.0766 & 0.001 \\
5 & 0.0509 & 0.0367 & 0.001 \\
6 & 0.0250 & 0.0272 & 0.0008 \\
7 & 0.0086 & 0.0088 & 0.0005 \\
8 & 0.0039 & 0.0037 & 0.0003 \\
9 & 0.0016 & 0.0017 & 0.0002 \\
10 & 0.0003 & 0.0004 & 0.00009 \\
11 & 0.00054 & 0.00021 & 0.00001 \\
12 & 0.00013 & 0.00013 & 0.00005 \\
\hline
\end{tabular}

Now we compare the speed advantage of the splitting method to the direct method for 8 line outages, an example of a mildly rare event. To do this, we change the number of samplings of the splitting method so that the variance of $p(8)$ estimated with the splitting method is the same as the variance of $p(8)$ estimated with the direct method.

For the direct method, Table I shows that $\hat{p}(8)=0.0039$ and the number of samples is $n=38000$. According to (15),

$$
\sigma^{2}(p(8)) \approx 1.02 \times 10^{-7} .
$$

For the splitting method, we choose $N_{1}=5886, N_{2}=4689$ and obtain $K_{1}=475, K_{2}=131$, and $N_{2}(8)=215$. Then, using (18), the variance of $p(8)$ for the splitting method in this set up is also given by (22).

This same variance was produced by $5886+4689 \approx 10600$ sample cascades of the splitting method and 38000 sampled cascades of the direct method. The ratio $R$ of the number of 


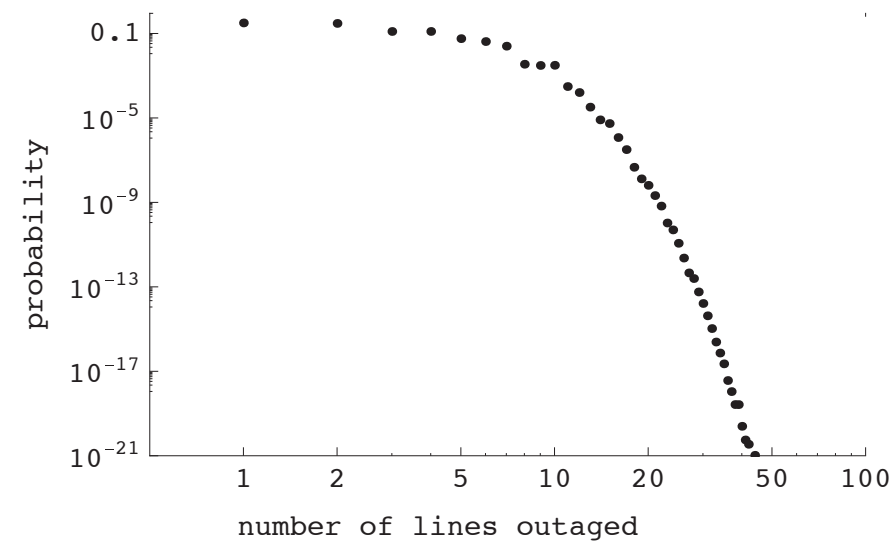

Fig. 5. Probability distribution of the number of line outages using the splitting method on the IEEE 225 bus system.

sampled cascades is about 0.3 . We conclude that the splitting method is faster than the direct method for estimating the probability of mildly rare events such as 8 line outages, but for these mildly rare events the speed advantage is not dramatic.

Finally, we note that the approximate formula (19) gives the similar results

$$
R=2\left(\frac{215}{475}+1\right) \frac{475}{5886}=0.23
$$

However, the approximate upper bound (20) is not effective for early stages such as $l=2$. The upper bound (20) becomes useful for $l \geq 3$.

\section{B. Simulating to reach 44 line outages with 11 stages}

We now show the splitting method estimating the very small probabilities of large numbers of line outages. Although rare events, these cascades are important because they correspond to large blackouts that have high impact on society [1]. To check the validity of the method on different systems, we use the IEEE 225 bus test system. This test shows the power of the splitting method to estimate the probability of rare cascading events. Indeed comparison with the direct method is impossible because the direct method is much too slow to be able to compute such rare events. The stages are $S_{0}=0, S_{1}=4, S_{2}=8, \ldots, S_{11}=44$ outages and there are $K_{i}=100$ samples for each stage $i$.

The probability to reach each stage is shown in Table II and the probability distribution of the number of outages is shown in Fig. 5. These results demonstrate that the splitting method can estimate probabilities of very rare cases such as 35 outages, which are inaccessible to direct methods.

\section{DISCUSSION OF FUTURE APPLICATIONS}

The splitting method is tested in this paper on a version of OPA with a fixed power grid, but our analysis of splitting is general and suggests that splitting could be generally applied to speed up cascading simulations that sample many cascades to determine cascading probabilities and risk. ${ }^{4}$ For simulations

\footnotetext{
${ }^{4}$ Note that the software modifications to implement splitting by storing outcomes of simulation stages and sampling from these to restart the simulation are straightforward.
}

TABLE II

PROBABILITY TO REACH NEXT STAGE

\begin{tabular}{cc} 
stage(failures) & probability \\
$1(4)$ & 0.25879 \\
$2(8)$ & 0.039401 \\
$3(12)$ & 0.020346 \\
$4(16)$ & 0.0075438 \\
$5(20)$ & 0.0059884 \\
$6(24)$ & 0.0068752 \\
$7(28)$ & 0.0050715 \\
$8(32)$ & 0.0042935 \\
$9(36)$ & 0.003792 \\
$10(40)$ & 0.0001896 \\
$11(44)$ & 0 \\
\hline
\end{tabular}

with a probabilistic framework that estimate cascading risk by repeated sampling in fixed power grids (for example, [10], [13], [27], [31], [40]), it seems clear that splitting could potentially offer substantial gains in performance. And there may be other calculations that splitting could speed up. For example, the random chemistry algorithm of [41] successively divides a large sample of initiating contingencies to find an unbiased minimal sample of multiple initiating contingencies that cause large blackouts. Simulation is used repeatedly to compute the cascading caused by the various initiating contingencies and could potentially be sped up using splitting. ${ }^{5}$ It may also be possible to use splitting to speed up methods of testing of controls to mitigate cascading, such as [42], [43]. In all these applications, the splitting method would play the limited and purely technical role of making cascade samples quicker to calculate, but the increase in speed could lead to a significant expansion of the range of applicability, particularly in enabling systematic sampling from all sources of variation and the use of larger grid models.

There are also applications in which it is not obvious how or whether splitting can be applied. For example, the complex systems dynamic version of OPA [14], [15] samples cascades from a slowly evolving grid, and it is not clear how the evolution should be integrated with the resampling for the splitting.

\section{Conclusion}

Simulation speed is a key barrier in assessing the risk of large cascading blackouts. We analyze the splitting method and test it on a standard power system simulation of cascading transmission line outages by estimating the probability mass function of the number of line outages. We show that the probability estimates are approximately maximum likelihood estimates and show how to design the optimal number of stages in the splitting method. While the splitting method has a modest speed advantage over direct Monte Carlo computation for estimating the probability of a small number of line outages, it is able to compute very rare samples of large numbers of line failures that correspond to the large blackouts of high risk to society. Direct Monte Carlo methods cannot be used to compute these rare samples because of their impractically excessive simulation times.

\footnotetext{
${ }^{5}$ The uniformity of sampling with splitting would be used but it would not be necessary to calculate the probabilities of the cascades.
} 
The splitting method is a very promising method for significantly improving the performance of cascading failure simulations. The splitting method is well aligned with and takes advantage of the structure of the cascading processes.

\section{REFERENCES}

[1] D. E. Newman, B. A. Carreras, V. E. Lynch, I. Dobson, Exploring complex systems aspects of blackout risk and mitigation, IEEE Trans. Reliability, vol. 60, no. 1, pp. 134-143, March 2011.

[2] NERC (North American Electric Reliability Council), 1996 system disturbances, (Available from NERC, Princeton Forrestal Village, 116-390 Village Boulevard, Princeton, New Jersey 08540-5731), 2002.

[3] D.N. Kosterev, C.W. Taylor, W.A. Mittelstadt, Model validation for the August 10, 1996 WSCC system outage, IEEE Trans. Power Systems, vol. 13, no. 3, pp. 967-979, 1999.

[4] U.S.-Canada Power System Outage Task Force, Final Report on the August 14th blackout in the United States and Canada. United States Department of Energy and National Resources Canada. April 2004.

[5] Task Force on Blackout Experience, Mitigation, and Role of New Technologies, of the IEEE PES Power System Dynamic Performance Committee, Blackout experiences and lessons, best practices for system dynamic performance, and the role of new technologies, IEEE Special Publication 07TP190, July 2007.

[6] IEEE PES CAMS Task Force on Cascading Failure, Initial review of methods for cascading failure analysis in electric power transmission systems, IEEE Power and Energy Society General Meeting, Pittsburgh PA USA, July 2008.

[7] B. A. Carreras, D. E. Newman, I. Dobson, and A. B. Poole, Evidence for self-organized criticality in a time series of electric power system blackouts, IEEE Transactions on Circuits and Systems Part I, vol. 51, no. 9, Sept. 2004, pp. 1733-1740.

[8] P. Hines, J. Apt, S. Talukdar, Large blackouts in North America: Historical trends and policy implications, Energy Policy, vol. 37, 2009, pp. 5249-5259.

[9] M. Papic, K. Bell, Y. Chen, I. Dobson, L. Fonte, E. Haq, P. Hines, D.Kirschen, X. Luo, S.S. Miller, N. Samaan, M. Vaiman, M. Varghese, P. Zhang, Survey of tools for risk assessment of cascading outages, IEEE Power and Energy Society General Meeting, Detroit MI USA, July 2011.

[10] I. Dobson, J. Kim, K. R. Wierzbicki, Testing branching process estimators of cascading failure with data from a simulation of transmission line outages, Risk Analysis, vol. 30, no. 4, 2010, pp. 650-662.

[11] H. Ren, I. Dobson, Using transmission line outage data to estimate cascading failure propagation in an electric power system, IEEE Trans. Circuits and Systems Part II, vol. 55, no. 9, Sept. 2008, pp. 927-931.

[12] I. Dobson, Estimating the propagation and extent of cascading line outages from utility data with a branching process, IEEE Transactions on Power Systems, vol. 27, no. 4, November 2012, pp. 2146-2155.

[13] B. A. Carreras, V.E. Lynch, I. Dobson and D. E. Newman, Critical points and transitions in a power transmission model, Chaos, vol 12, no 4, December 2002, pp. 985-994.

[14] B.A. Carreras, V.E. Lynch, I. Dobson, and D.E. Newman, Complex dynamics of blackouts in power transmission systems, Chaos, vol. 14, no. 3, September 2004, pp. 643-652.

[15] H. Ren, I. Dobson, and B.A. Carreras, Long-term effect of the n-1 criterion on cascading line outages in an evolving power transmission grid, IEEE Transactions on Power Systems, vol. 23, no. 3, August 2008, pp. 1217-1225.

[16] M. Villen-Altamirano, J. Villen-Altamirano, RESTART: A method for accelerating rare event simulations, Proceedings of 13th Int. Teletraffic Congress, Queueing, Performance and Control in ATM, ed. J.W. Cohen, 1991.
[17] F. Schreiber, C. Gorg, Rare event simulation: A modified RESTART method Using the LRE-Algorithm, Proceedings 1996 Winter Simulation Conference, Coronado, CA USA 1996.

[18] M. Villen-Altamirano, J. Villen-Altamirano, Enhancement of the accelerated simulation method RESTART by considering multiple thresholds, Proceedings of 14th Int. Teletraffic Congress, The Fundamental Role Of Teletraffic in the Evolution of Telecom. Networks, eds. J. Labetouille, J.W. Roberts, 1994.

[19] M. Villen-Altamirano, J. Villen-Altamirano, On the efficiency of RESTART for multi-dimensional systems, ACM Trans. Model. Comput. Simul., vol. 16, no. 3, pp. 251-279, 2006.

[20] P. Glasserman, P. Heidelberger, P. Shahabuddin, T. Zajic, A large deviations perspective on the efficiency of multilevel splitting, IEEE Trans. Automatic Control, vol. 43, no. 12, Dec. 1998, pp. 1666-1679.

[21] A. Dubi, General statistical model for geometrical splitting in Monte Carlo-I, Transport Theory and Statistical Physics, Vol. 14, pp. 167-193, 1985.

[22] A.V. Starkov, Monte Carlo splitting importance sampling, Monte Carlo Methods and Applications, vol.1, pp. 241-250, 1995.

[23] J. F. Shortle, C.-H. Chen, A preliminary study of optimal splitting for rare-event simulation, Proceedings 2008 Winter Simulation Conference, Miami FL USA, December 2008.

[24] S.-P. Wang, A. Chen, C.-W. Liu, C.-H. Chen, J. F. Shortle, Rare-event splitting simulation for analysis of power system blackouts, IEEE Power and Energy Society General Meeting, Detroit MI USA July 2011.

[25] J. F. Shortle, Efficient simulation of blackout probabilities using splitting, Electrical Power and Energy Systems, vol. 44, 2013, pp. 743-751.

[26] J. Kim, Quantifying failure propagation in electric power transmission systems, PhD thesis, University of Wisconsin, Madison WI 53706 USA, 2011.

[27] J. Chen, J.S. Thorp, I. Dobson, Cascading dynamics and mitigation assessment in power system disturbances via a hidden failure model, International Journal Electrical Power and Energy Systems, vol. 27, no. 4, May 2005, pp. 318-326.

[28] J.A. Bucklew, P. Ney, J.S. Sadowsky, Monte Carlo simulation and large deviations theory for uniformly recurrent Markov chains, Journal of Applied Probability, vol. 27, pp. 44-59, March 1990.

[29] R. K. Bahr, J. A. Bucklew, Quick simulation of detector error probabilities in the presence of memory and non-linearity, IEEE Trans. Communications, Vol. 41, No. 11, pp. 1-7, Nov. 1993.

[30] J.A. Bucklew, J.A. Gubner, Bias point selection in the importance sampling Monte Carlo simulation of systems, IEEE Trans. Signal Processing, Vol. 51, No. 1, pp. 152-159, January 2003.

[31] D.S. Kirschen, D. Jawayeera, D.P. Nedic, R.N. Allan, A probabilistic indicator of system stress, IEEE Trans. Power Systems, vol. 19 , no. 3, 2004, pp. 1650-1657.

[32] B.A. Carreras, D.E. Newman, B.A. Carreras, I. Dobson, N.S. Degala, Validating OPA with WECC data, Forty-sixth Hawaii International Conference on System Sciences, Maui, Hawaii, January 2013.

[33] T.E. Harris, Theory of branching processes, Dover NY 1989.

[34] K.B. Athreya, P.E. Ney, Branching processes, Dover NY 2004 (reprint of Springer-verlag Berlin 1972).

[35] I. Dobson, B.A. Carreras, D.E. Newman, A loading-dependent model of probabilistic cascading failure, Probability in the Engineering and Informational Sciences, vol. 19, no. 1, 2005.

[36] J. Kim, I. Dobson, Approximating a loading-dependent cascading failure model with a branching process, IEEE Trans. Reliability, vol. 59, no. 4, December 2010, pp. 691-699.

[37] J.A. Bucklew, An introduction to rare event simulation, Springer Series in Statistics, 2004.

[38] M. Garvels, A comparison of RESTART implementations, Proceedings of 1998 Winter Simulation Conference, 1998. 
[39] P. L'Ecuyer, V. Demers, Rare events, splitting, and quasi-Monte Carlo, ACM Trans. Model. and Comp. Simul., Vol. 17, No. 2, Article 9, April, 2007.

[40] Quan Chen and L. Mili, Risk-based composite power system vulnerability evaluation to cascading failures using importance sampling, Power and Energy Society General Meeting, Detroit, MI USA 2011.

[41] M. J. Eppstein and P. D. H. Hines, A "random chemistry" algorithm for identifying multiple contingencies that initiate cascading failure, IEEE Transactions on Power Systems, vol. 27, no. 3, August 2012, pp.1698-1705.

[42] R. Pfitzner, K. Turitsyn, and M. Chertkov, Controlled tripping of overheated lines mitigates power outages, 2011, arXiv:1104.4558 [physics.soc-ph]

[43] A. Bernstein, D. Bienstock, D. Hay, M. Uzunoglu, and G. Zussman, Power grid vulnerability to geographically correlated failures - analysis and control implications, 2012 arXiv:1206.1099 [cs.SY]

Janghoon Kim (S 05, M 12) received the BS degree in electrical power systems from Seoul National University, Seoul, Korea in 2002. He worked for Sewoo Motors from 2002 to 2005. He received the MS and PhD degrees in electrical engineering from the University of Wisconsin-Madison, USA in 2008 and 2011. He joined Samsung Corporation in 2012.

Ian Dobson (F 06) received the BA degree in Mathematics from Cambridge, England in 1978 and the $\mathrm{PhD}$ degree in Electrical Engineering from Cornell University USA in 1989. He is currently Sandbulte professor of engineering at Iowa State University USA.

James A. Bucklew (F 04) received the Ph.D. degree in electrical engineering from Purdue University, West Lafayette, IN, in 1979. He is currently a Professor with the Department of Electrical and Computer Engineering and the Department of Mathematics at the University of Wisconsin, Madison. His research interests include the application of probability and statistics to signal processing and communication problems. He has published over 100 articles in these fields. He has also published several fly fishing articles (in Spanish) and also works as a translator. He is the author of the books Large Deviation Techniques in Decision, Simulation, and Estimation (WileyInterscience 1990), and Introduction to Rare Event Simulation (SpringerVerlag, 2004). Dr. Bucklew has served as an Associate Editor (1990-1992) for IEEE Transactions on Information Theory and as an Associate Editor (1997-1999) for the IEEE Transactions on Signal Processing. 